\title{
Quality of Experience Based Resource Sharing in IEEE 802.11e HCCA
}

\author{
Jorge Navarro-Ortiz, Juan M. Lopez-Soler \\ Dept. of Signal Theory, Telematics and Communications \\ University of Granada \\ Granada, Spain \\ jorgenavarro@ugr.es; juanma@ugr.es
}

\author{
Giovanni Stea \\ Dipartimento di Ingegneria dell'Informazione \\ University of Pisa \\ Pisa, Italy \\ g.stea@iet.unipi.it
}

\begin{abstract}
When dealing with multiple traffic classes, it is often difficult to define a single resource sharing criterion. For instance, VoIP flows require low delay and losses, whereas FTP traffic requires high throughput. In this paper, we propose to use the Mean Opinion Score (MOS) as a unifying criterion, and to share resources so as to keep the MOS of the various flows above an acceptable threshold whenever possible. We present four different resource sharing policies: Preemptive, Non-preemptive, MOS-driven Fairness and Revenue Optimization. We analyze the different approaches through simulation, comparing their performance in terms of end-user Quality of Experience and revenue. Our results show that our scheme reacts to the changing traffic scenarios so as to achieve the pre-specified objective, thus achieving an effective exploitation of the limited bandwidth resources.
\end{abstract}

Keywords-802.11e; HCCA; Quality of Experience; Scheduling; Resource allocation policies

\section{INTRODUCTION}

In this paper we present resource sharing algorithms for multiple classes of service, suitable for a WLAN employing 802.11e Hybrid Coordination Function Controlled Channel Access (HCCA). The latter is defined in the IEEE 802.11e standard [1], together with the Enhanced Distributed Channel Access (EDCA). While EDCA differentiates among the different traffic classes using relative priorities, HCCA supports strict QoS requirements by means of a centralized packet scheduler at the access point (AP). HCCA can be implemented on a standard 802.11e card via software drivers [2].

While the literature on packet scheduling for HCCA (and, more in general, for WLANs) is abundant [3][4][5], a comparatively minor attention has been devoted to how to configure schedulers for supporting a given traffic mix. In fact, while a scheduler enforces a given resource sharing, by ensuring that every Traffic Class (TC) gets no more than its reserved bandwidth in time of congestion, how to configure the latter to achieve a good resource utilization is often left to the (heuristic) network administrator know-how. A static resource sharing policy, where each TC gets a fixed amount of bandwidth, would clearly be suboptimal, as it would not cope with the dynamic evolution of the traffic: as flows come and go, the bandwidth required by a TC might fluctuate sensibly. A possible way to implement a dynamic resource sharing would be to have flow setup/teardown protocols reconfigure the scheduler, so as to modify its bandwidth sharing properties according to the traffic demands. Such protocols, however, work above the IP layer: having an AP manage them would complicate its hardware. For this reason, they are seldom - if ever - supported.

As bandwidth sharing criteria should be driven by performance, a problem closely related to the former is how to define a common performance metric in a multi-class environment. For instance, VoIP traffic requires low delay and losses, whereas TCP traffic requires a stable bandwidth; FTP, which run on TCP, requires high throughput.

In this paper, we propose to employ a unified Quality of Experience (QoE) performance metric, i.e. the Mean Opinion Score (MOS), as a unifying criterion for bandwidth sharing. We define ways to compose measurable performance metrics in order to compute a MOS for several TCs (conversational, video streaming, TCP real-time, interactive and background), also drawing from the existing literature on the subject. Capitalizing on that, we propose dynamic resource sharing policies, which react to the changing traffic load, trying to optimize the MOS of each TC when possible. More specifically, we propose policies that allow a network administrator to customize the way that transmission opportunities (TXOPs) are granted in HCCA. We describe four of them: Preemptive, Non-preemptive, MOS-driven Fairness and Revenue Optimization, highlighting their differences and assessing their performance via simulation.

To the best of our knowledge, there is no resource allocation method in the current literature of 802.11e networks to share resources among different TCs or services. However, other wireless technologies such as UMTS (UMTS QoS classes [6]) or LTE (QoS Class Identifier [7]) utilize this concept to control bearer level packet forwarding treatment (e.g. scheduling weights, admission thresholds, queue management thresholds, etc.). A few works ([8]-[11]) actually advocate using the MOS for allocating resources inside a network. In fact, they propose solving a cross-layer optimization problem, whose objective is to optimize the sum of the MOS for a set of users. The latter is assumed to be a non linear function of the data rate, and the analysis requires continuous adaptive-rate applications, which leaves out all real-time applications such as VoIP, which are intermittent and/or non-adaptive. Moreover, no Radio Resource Management (RRM) algorithm (scheduler, admission control,

The research in this work has been partially supported by the Ministerio de Ciencia e Innovación of Spain (project TIN2009-13992-C02-02). 
etc.) for HCCA has based its online decisions on subjective quality metrics such as MOS. Nevertheless, this approximation is not new in other radio technologies such as OFDMA. Some proposals [12] utilize utility functions to maximize the aggregate of the subjective quality experienced by the end-user.

The rest of this paper is organized as follows. Section 2 describes the traffic classes and services utilized in our work, as well as the procedures to estimate their subjective quality. The proposed resource sharing policies are found in Section 3. Section 4 describes the simulation results and conclusions are drawn in Section 5.

\section{TRAFFIC Classes AND PERFORMANCE METRICS}

Traffic classes (TC) define a set of services with similar QoS requirements (extended from UMTS [6]). We adopted the following classification:

- Conversational - preserve time relation between packets; conversational pattern (stringent and low delay).

- $\quad$ Streaming - preserve time relation between packets.

- $\quad$ TCP real-time - similar to the above, but using TCP as a transport protocol.

- Interactive - request response pattern; preserve payload content.

- Background - the destination is not expecting the data within a certain time; preserve payload content.

In our analysis, the following services were considered: Voice over IP (VoIP) for Conversational, RTP/UDP video streaming for Streaming, TCP video streaming (e.g., YouTube video broadcasting) for $T C P$ real-time, web browsing for Interactive and FTP for Background.

The end-user experience depends on the specific traffic service. Accordingly, for QoE-based bandwidth sharing among multiple services, a unifying criterion must be defined. Hereafter, we show how to compute an estimate of a subjective user perception using objective quality metrics for each TC.

\section{A. Quality Metric Functions}

This section describes the quality metric functions for the different TCs used in this paper. These functions map objective network metrics (such as delay or throughput) into subjective quality. Following [13], we call it Mean Opinion Score (MOS). The MOS is a continuous function ranging from 1 (all users dissatisfied) to 5 (all users satisfied).

VoIP: The MOS estimation for VoIP is based on the E-model [14], whose formula is in Eq. 1. For our evaluation, an indoor wireless scenario was simulated using G.711 codec. The parameters of Table I were assumed.

$$
\text { MOS }=\left\{\begin{array}{c}
1.0 \text { if } R<0 \\
4.5 \text { if } R>100 \\
1+3.5 \cdot 10^{-3} R+7 \cdot 10^{-6} R \cdot(R-60) \cdot(100-R) \text { otherwise }
\end{array}\right.
$$

where

$$
\begin{gathered}
R=R_{0}-I_{s}-I_{d}-I_{e, e f f}+A \\
I_{d}=0.024 \cdot D+\max \{0,0.11 \cdot(D-177.3)\}
\end{gathered}
$$

$$
I_{e, e f f}=I_{e}+\frac{\left(95-I_{e}\right) \times l p}{l p+B_{p l}}
$$

$l p$ is the loss probability and $D$ is the delay in milliseconds.

Video streaming: The subjective quality evaluation for video streaming is based on the EvalVid framework [15], which evaluates the differences between the original and the resulting images after network transmission. However, due to the image reconstruction process, it cannot be used for online algorithms, but only for offline quality estimations.

In [16], two learning models were described for online video quality prediction using EvalVid. Based on the temporal (movement) and spatial (edges, brightness) features, videos are classified into three groups: slight movement (SM), gentle walking $(\mathrm{GW})$ and rapid movement $(\mathrm{RM})$. The video quality, based on the Frame Rate (FR, fps), Sending Bit Rate (SBR, kbps) and Packet Error Rate (PER), is given by:

$$
M O S=\frac{a_{1}+a_{2} F R+a_{3} \ln (S B R)}{1+a_{4} P E R+a_{5}(P E R)^{2}}
$$

The $a_{i}$ coefficients for SM, GW, RM are shown in Table II.

TCP video: TCP video is buffered at the receiver according to a dual-threshold strategy [17]. The first threshold (BTT, buffered time threshold) defines the minimum buffer size (in time units) before playout starts. The second threshold defines the buffer size at which the receiver signals the server to stop sending data.

Due to the TCP reliable delivery, subjective quality of TCP video streaming can be mostly affected by buffer underruns, which interrupt the playout process. Since buffer underruns are caused by short-term variations, an instantaneous quality metric estimate cannot be obtained from user surveys that last tens or hundreds of seconds. Therefore, a simplified artificial MOS function has been designed which depends only on the instantaneous buffer conditions. The criteria for designing the function are:

- The incurred delay due to initial buffering is usually expected and thus it should not decrease the MOS.

- Buffer underruns and rebuffering shall abruptly reduce MOS to 1 since they halt the playout, which is annoying.

- After an underrun, MOS should not increase rapidly. This reflects that after a video interruption, it takes some time

TABLE I. E-MOdEL PARAMETERS FOR INDOOR WITH G.711 CODEC

\begin{tabular}{|l|l|l|}
\hline Parameter & Value & \multicolumn{1}{c|}{ Description } \\
\hline $\mathrm{A}$ & 5 & Advantage factor \\
\hline $\mathrm{R}_{0}$ & 93.2 & Basic signal-to-noise ratio \\
\hline $\mathrm{I}_{\mathrm{S}}$ & 0.0 & Simultaneous impairment factor \\
\hline $\mathrm{I}_{\mathrm{e}}$ & 0.0 & Equipment impairment factor \\
\hline $\mathrm{B}_{\mathrm{pl}}$ & 0.043 & Packet-loss robustness factor \\
\hline
\end{tabular}

TABLE II. $\quad A_{l}$ DEFINITIONS FOR Video STREAMING MOS (EQ. 2)

\begin{tabular}{|l|l|l|l|}
\hline Coefficient & \multicolumn{1}{|c|}{ SM } & \multicolumn{1}{|c|}{ GW } & \multicolumn{1}{|c|}{ RM } \\
\hline $\mathrm{a}_{1}$ & 4.5796 & 3.4757 & 3.0946 \\
\hline $\mathrm{a}_{2}$ & -0.0065 & 0.0022 & -0.0065 \\
\hline $\mathrm{a}_{3}$ & 0.0573 & 0.0407 & 0.1464 \\
\hline $\mathrm{a}_{4}$ & 2.2073 & 2.4984 & 10.0437 \\
\hline $\mathrm{a}_{5}$ & 7.1773 & -3.7433 & 0.6865 \\
\hline
\end{tabular}


before the user returns to be satisfied again. Besides, this approach avoids unwanted MOS oscillations.

- If the buffer level gets below the first threshold, then the MOS should decrease, albeit not abruptly, so as to capture the likelihood that the buffer will underrun.

Fig. 1 shows an example of the desired behavior. The MOS function for TCP video streaming is given in Eq. 3 and 4.

$$
\begin{gathered}
\operatorname{MOS}[k]=\left\{\begin{array}{c}
5 \text { if } B T \geq B T T \\
1+(5-1) \times B T / B T T \text { if } 0<B T<B T T \\
1 \text { if } B T \leq 0
\end{array}\right. \\
\operatorname{AVGMOS}[k]=\left\{\begin{array}{c}
\operatorname{MOS}[k] \text { if } \operatorname{MOS}[k]<A V G M O S[k-1] \\
\propto \text { AVGMOS }[k-1]+(1-\propto) \text { MOS }[k] \text { otherwis }
\end{array}\right.
\end{gathered}
$$

where $B T$ is the buffered time, i.e. playout time corresponding to the packets in the receiver buffer.

The $\alpha$ parameter allows the network administrator to adjust the end-user impairment after a buffer underrun. We define the convergence time $(C T)$ as the time elapsing from a complete rebuffering until the user is totally satisfied again (i.e., $M O S=$ $5-\varepsilon$ ). This time depends on $\alpha$ as follows:

$$
\propto=\sqrt[\left(\frac{C T}{M F P}\right)]{\varepsilon / 4}
$$

where MFP is the MOS Filtering Period (defined in Section 3) in seconds. As an example, $\alpha=0.48$ implies $C T=5 \mathrm{~s}$, with $M F P$ $=1 \mathrm{~s}$ and $\varepsilon=0.1$.

Web browsing: The MOS function was obtained after a survey [12] during which 52 volunteers were asked to rate the download of 14 different web pages at different application data rates (from few kbps to $2 \mathrm{Mbps}$ ). Eq. 6 shows the relationship between throughput and MOS, assuming an average web page size of 130 Kbytes [18].

$$
M O S=5-\frac{578}{1+\left(\frac{T H R+541.1}{45.98}\right)^{2}}
$$

where $T H R$ is the throughput in kbps.

FTP: In [19], Y. Sato et al studied user's subjective assessment of communication quality in bulk data transmission. They surveyed 91 users, considering typical file sizes and FTTH-like bandwidths (max. $25 \mathrm{Mbps}$ ).

For our purposes, we scaled the maximum throughput to take into account IEEE 802.11e's scarcer bandwidth. Based on IEEE $802.11 \mathrm{~b}$ physical layer, we defined $1 \mathrm{Mbps}$ as the target

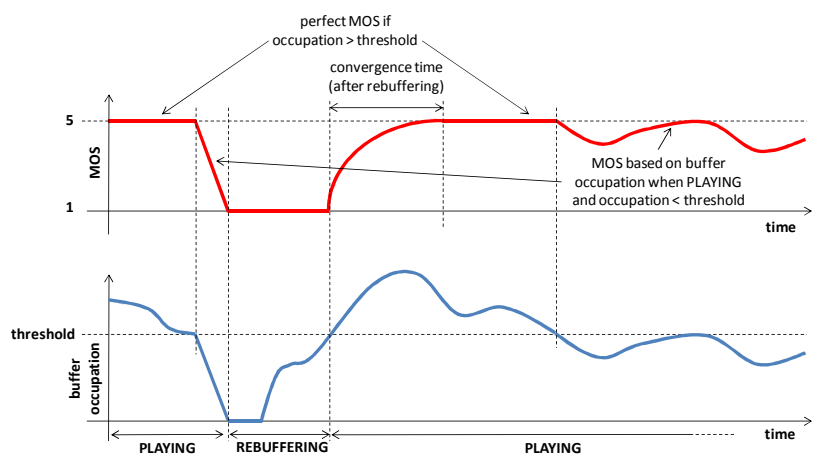

Figure 1. Artificial MOS evolution for TCP video streaming. throughput for the FTP service, thus scaling the results by a factor of 25. With these assumptions, MOS is computed as:

$$
\text { MOS }=6.5 \times T H R-0,54
$$

where $T H R$ is the throughput measured in Mbps.

\section{MOS-BASED RESOURCE SHARING POLICIES FOR HCCA}

In [20] we presented a dynamic resource allocation algorithm, the territory method, whose objective is maximizing the resource utilization while maintaining end-user satisfaction. In addition, it aims at prioritizing real-time services and at improving the performance of TCP applications.

This method shares the common pool of transmission opportunities (TXOPs) into different territories (see Fig. 2), one per each TC. Each territory has a customizable minimum capacity $(M C)$, which can be non null to avoid starvation. A higher reserved capacity $(R C)$ can be harvested by booking from a pool of the spare resources. The RC fluctuates according to the end-user MOS, as shown in Fig. 3. This resource allocation algorithm was designed so that the average MOS of certain TC (average of MOS values from the different flows belonging to that TC) is expected to stay between an upper MOS threshold (UMT) and a lower MOS threshold (LMT). If the estimated MOS is smaller than the LMT, the end-user perception is worse than required and $\mathrm{RC}$ will increase in order to keep the MOS within the required range. On the contrary, if it is above the UMT, the end-user experience is better than required and hence part of the $\mathrm{RC}$ can be returned. Both MOS measurements and territory updates are performed at a customizable MOS Filtering Period (MFP). At the AP, a scheduler (the Monolithic Shaper Scheduler, MSS [21]) arbitrates the TCs according to these territories. First, the territory method checks against a round-robin list which TC owns the current TXOP. Then, the scheduler selects a flow belonging to that TC for packet transmission. As long as the scheduler enforces a fair queueing, the actual scheduling policy has a minor impact on the results.

The resource sharing policy described in [20], being a first attempt to define a resource sharing policy in $802.11 \mathrm{e}$, needs to be adapted to dynamically varying traffic. First of all, a flexible and effective algorithm for updating the territory has to be devised. The latter should take into account the different dynamics of the various TCs (e.g., web traffic is bursty and

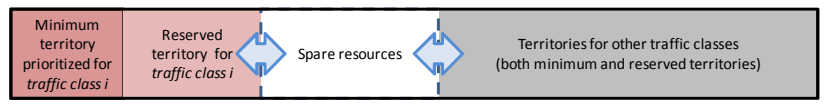

Figure 2. Example of territories definition for a generic traffic class.

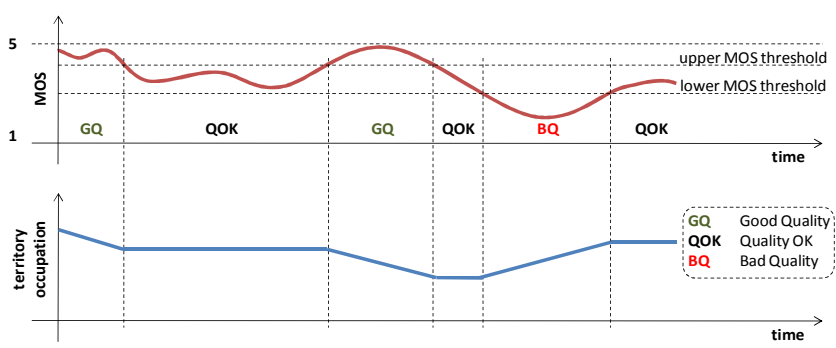

Figure 3. Example of the relationship between subjective quality and the territory evolution for a generic traffic class. 
intermittent, hence requiring fast territory modifications, whereas FTP is continuous, which calls for smoother variations). Furthermore, only a preemptive resource sharing policy was considered in [20]: TCs were sorted by priority, and a higher priority $\mathrm{TC}$ could preempt the $\mathrm{RC}$ of a lower priority TC (although without eating into its MC). Accordingly, real-time services (conversational and streaming) were favored at the expenses of non real-time services (interactive and background).

The first enhancement is to use an exponential filtering when measuring a connection's average MOS, so as to avoid oscillations in the territory due to sheer instantaneous variations in the MOS. The averaging process is shown in Eq. 8.

$$
\operatorname{AVGMOS}_{i}[k]=\propto \cdot \operatorname{AVGMOS}_{i}[k-1]+(1-\alpha) \cdot \operatorname{MOS}_{i}[k]
$$

where $A V G M O S_{i}[k]$ is the average MOS of the traffic class $i$ for the $k$-th MOS filtering period, $\alpha$ is the averaging weight and $\operatorname{MOS}_{i}[k]$ is the MOS sample of the $k$-th period.

In addition, each $\mathrm{TC}$ can select its own averaging period depending on its traffic characteristics. For example, services with stable traffic generation (e.g. FTP) could have larger periods than services with bursty traffic (e.g. web browsing). In the latter case the traffic pattern varies more quickly, and long periods could make territories not to react properly to those changes.

Furthermore, we customized the epochs for adjusting the territories. The current implementation considers different Territory Update Periods (TUP), one per TC, with the objective of considering their different properties. Several MOS samples should be considered for resizing the territories: too few samples might lead to fluctuations in the territories due to MOS peaks, hence leading to unstable performance of the services; too many samples might lead to unresponsiveness, again causing a suboptimal performance. We have selected 5 MOS samples for averaging by default, i.e. the territory update periods are equal to $5 \mathrm{~s}$ for all TCs excluding interactive, whose period is $1 \mathrm{~s}$.

Another decision point regards how to increment/decrement the territories when LMT/UMT violations are detected. Again, responsiveness has to be traded for stability. Selecting the same step for both increasing and decreasing e.g. (1 out of 40 TXOPs, as in [20]), leads to unresponsiveness against e.g. a rapid traffic growth. A better result can be obtained by keeping the decrease step (DS) and the increase step (IS) different. The IS is selected proportional to the distance to the good quality range (MOS between LMT and UMT), i.e. it is larger if the MOS is very far from the requirements and smaller if it is near the UMT.

Eq. 9 illustrates how the IS is calculated for a territory update period. The maximum step has been set to $20 \%$ of the resources. For the DS a fixed value of 1 TXOP was chosen, lest a drastic decrease leads to performance degradation.

$$
I S=\left\lceil\text { max_step } \times \frac{\text { current_MOS_diff }]}{\text { max_MOS_diff }}\right\rceil
$$

where

$$
\text { max_MOS_diff }=\left(\frac{U M T+L M T}{2}-1\right)
$$

$$
\text { current_MOS_diff }=\left(\frac{U M T+L M T}{2}-A V G M O S\right)
$$

The following subsections describe different policies for resource sharing in IEEE 802.11e networks based on the territory method.

Preemptive. This is the original policy in the territory method depicted in [20]. Its objective is to ensure that the performance of real-time services is not affected by non real-time traffic. For that purpose, higher priority traffic classes can preempt resources from the reserved capacity of lower priority TCs. Therefore, conversational services can always utilize all the transmission opportunities except those belonging to the minimum capacity of other traffic classes.

Fig. 4 describes the flowchart of this approach. For each TC $i$, the MOS is computed by averaging the MOS of the single constitutive flows, which are sampled at fixed intervals.

Non-preemptive. This approach assumes that no traffic class has priority over other TCs. Therefore, no spare resources can be pre-empted once another TC has got hold of them.

However, a certain service is guaranteed for each traffic class by means of its minimum capacity, which can be customized by the administrator.

This policy allocates resources based on the current needs of each TC. If the global MOS of a TC (average MOS of all flows belonging to that traffic class) is below its LMT, its territory will grow if there are spare resources. Similarly, if the average MOS is above the UMT part of the reserved resources will be released.

Thus, it is a first come, first served policy without priorities between traffic classes. Its flowchart is similar to Fig. 4 but substituting the "no spare resources" box with Fig. 5.

MOS-driven Fairness. Neither of the previous schemes can ensure inter-TC fairness. A MOS-driven Fairness policy behaves similarly to the Non-preemptive policy as long as the MOS of all the different TCs is good. However, it will try to equalize the MOS among TCs when there are not enough resources. In that situation, the TCs with the lowest MOS can preempt some RC from the TCs with the highest quality. In this case, the "no spare resources" box is shown in Fig. 6.

Revenue Optimization. Similarly, the Revenue Optimization approach only operates when the MOS of a TC is below its threshold. In that case, TCs are ranked by Revenue Efficiency (RE), i.e. income per resource unit (Eq. 10). The latter is computed by dividing the overall MOS of TC $i$ by the amount of resources used to support it. The result is weighted by a TCspecific revenue weight $W_{i}$, which can be selected by a network administrator to reflect his revenue policies. A higher revenue TC can preempt spare resources from a lower revenue TC (see Fig. 7).

$$
R E_{i}=W_{i} \cdot\left(A V G M O S_{i}-1\right) / R C_{i}
$$

\section{PERFORMANCE EVALUATION}

The performance of the above-defined policies was evaluated via simulation. Section 4.1 introduces the simulation setup. Section 4.2 describes the traffic generation. Simulation 


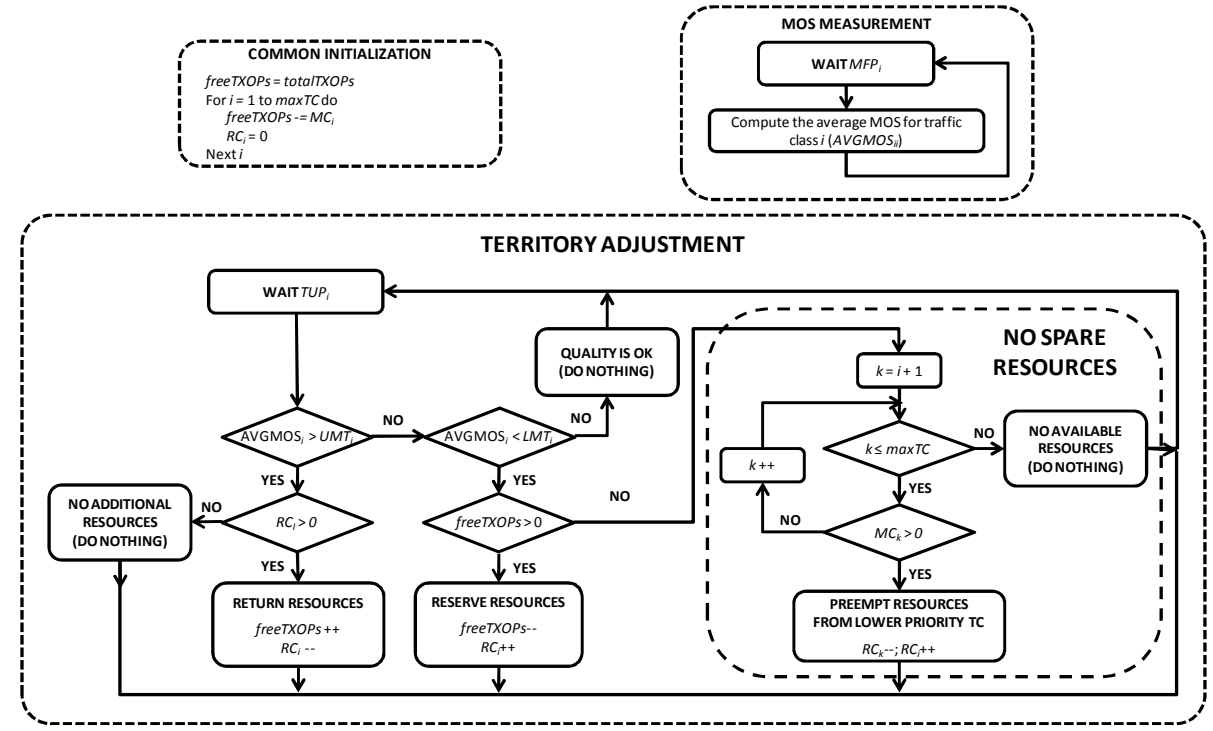

Figure 4. Flowchart of the territory method using the Preemptive policy.
TABLE III. WIRELESS Physical Layer Parameters

\begin{tabular}{|c|c|c|c|}
\hline Parameter & Value & Parameter & Value \\
\hline SIFS & $10 \mu \mathrm{s}$ & $\begin{array}{c}\text { PHY header } \\
\left(\mathrm{h}_{\mathrm{PHY}}\right)\end{array}$ & 192 bits \\
\hline PIFS & $30 \mu \mathrm{s}$ & $\begin{array}{c}\text { MAC header } \\
\left(\mathrm{h}_{\mathrm{MAC}}\right)\end{array}$ & 288 bits \\
\hline DIFS & $50 \mu \mathrm{s}$ & $\begin{array}{c}\text { ACK length } \\
\left(\mathrm{h}_{\mathrm{ACK}}\right)\end{array}$ & 112 bits \\
\hline Slot Time & $20 \mu \mathrm{s}$ & $\begin{array}{c}\text { CF-POLL } \\
\text { length }\left(\mathrm{h}_{\mathrm{POLL}}\right)\end{array}$ & 288 bits \\
\hline Basic rate & $1 \mathrm{Mbps}$ & Data rate & $11 \mathrm{Mbps}$ \\
\hline
\end{tabular}

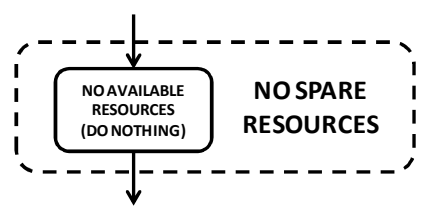

Figure 5. Flowchart for the Non-preemptive policy. results are analyzed in Section 4.3.

\section{A. Simulation setup}

Our simulations were carried out using $n s 2$ [22], together with some additional software modules. We employed the HCCA framework for ns2 described in [23]. The HCCA scheduler employed was the Monolithic Shaper-Scheduler (MSS), adapted to HCCA as explained in [20]. The policies described in Section 3 were implemented as part of the territory method.

A heterogeneous wired-cum-wireless scenario is simulated, where an AP is connected to several stations and to a wired part. Communications take place between wireless stations and servers or peers in the wired part.

The wireless physical layer is the High Rate Direct Sequence Spread Spectrum (HR-DSSS), also known as $802.11 \mathrm{~b}$, whose parameters are summarized in Table III.

Links in the wired part have high rate (100 Mbps) and low latency ( $2 \mathrm{~ms})$, so as to have a negligible impact on the results. Each minimum capacity (for the different TCs) in the territory

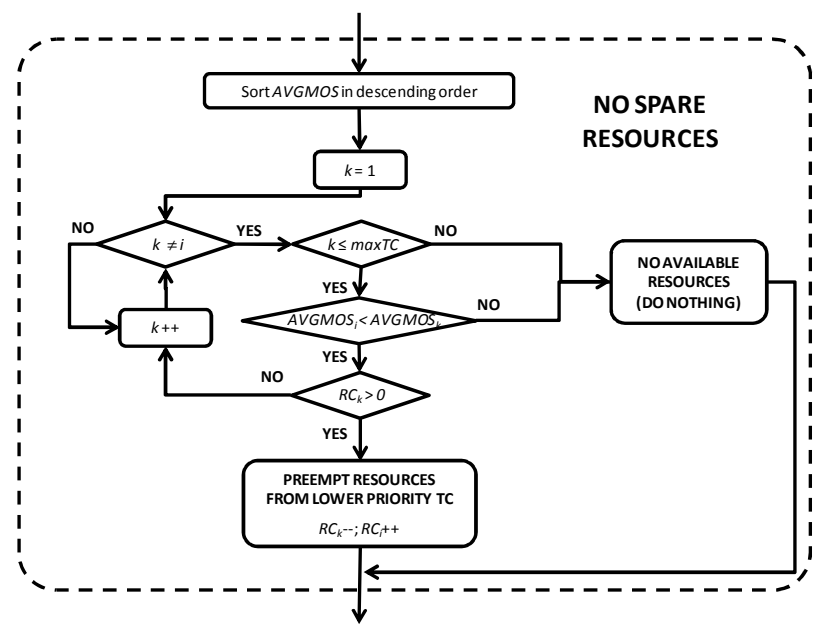

Figure 6. Flowchart for the MOS-driven Fairness policy. method has been set to $10 \%$ of the total capacity.

ANSWER [24] was used for automating the simulation experiments. In our simulations the $95 \%$ confidence intervals were computed over a maximum of 10 replicas per scenario.

\section{B. Traffic generation}

This section describes how the different services generate data for our performance evaluation.

VoIP traffic was generated according to an on-off pattern [25] [26], with on and off times consistent with bidirectional conversations. The G.711 codec is employed due to its wide diffusion in VoIP systems, packing three voice frames into a UDP/RTP packet ( 240 bytes +40 -byte header), i.e. to have an interpacket sending period of $30 \mathrm{~ms}$.

Video streaming traffic was generated using EvalVid [15], which supports trace file generation of MPEG-4, H.263 and H.264 video. It can be interfaced to $n s 2$ to generate traffic using real traces [27].

We selected a QCIF video sequence (176x144) encoded in

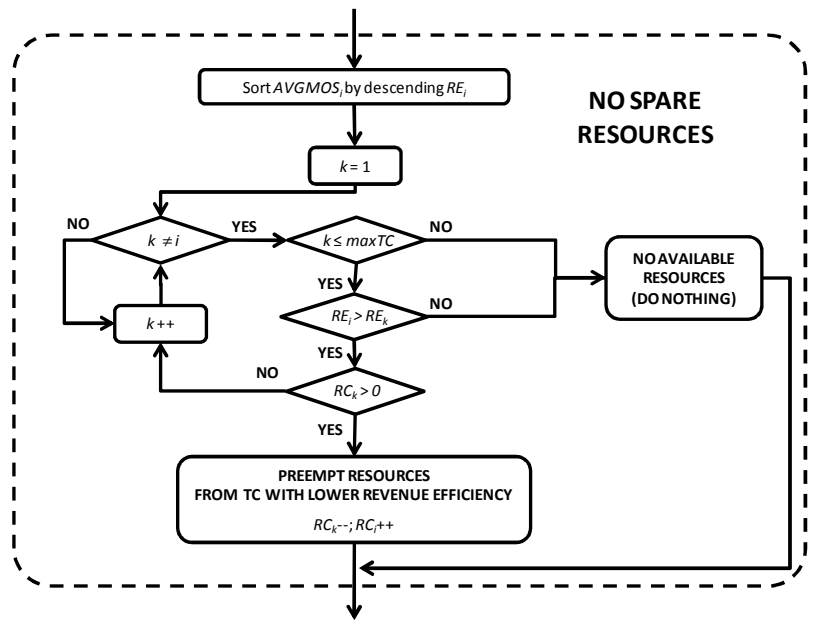

Figure 7. Flowchart for the Revenue Optimization policy. 
MPEG4 format following the description in [16]. We used the football video trace, which is a Rapid Movement one according to [16]. Variable Bit Rate traffic was generated at $15 \mathrm{fps}$, with an average frame size of 9445 bytes.

A playout buffer was implemented at the receiver side in order to mitigate the jitter. In our simulations, packets were discarded after 5s (default buffer size in Microsoft ${ }^{\circledR}$ Windows Media $^{\mathrm{TM}}$ Player) since buffering in the receiver.

TCP video streaming traffic was generated assuming the characteristics of YouTube's videos [28], i.e. a Constant-BitRate (CBR) traffic source with a bit-rate of $330 \mathrm{kbps}$. The threshold (BTT) of the playout buffer was set to $5 \mathrm{~s}$.

Web browsing. Our web traffic generator models page sizes as an exponential random variable, whose average is 130 Kbytes [18]. The user reading time is also exponentially distributed with an average of 61.73s [29].

$\boldsymbol{F T P}$ traffic was generated assuming infinite file sizes at the servers, so as to have always packets ready for transmission.

\section{Performance evaluation results}

We performed static simulations, i.e. a constant number of sources along the whole simulation. Different traffic loads were simulated so that the increase of VoIP sources drives the transition from good performance (in terms of subjective quality) to bad performance. This way, the different resource sharing policies can be seen in action while managing scarce resources. With this objective, the traffic was generated using 2 video streams, 3 TCP video streams, 5 web browsing sessions and 3 FTP downloads. VoIP calls varied from 0 to 30.

Preemptive policy: Fig. 8 shows the metrics for the Preemptive scheme as a function of the number of VoIP QoS stations (VoIPQSTAs), including MOS, the resource sharing (TXOPs percentage) for the considered traffic classes and the revenue metric $(R$, defined by Eq. 11$)$ per each TC and aggregated.

$$
R_{i}=W_{i} \cdot\left(\text { AVGMOS }_{i}-1\right)
$$

The territory distribution (Fig. 8b) shows that conversational class (VoIP) utilizes as many resources as needed, more as its traffic load increases. When conversational services do not reserve all the spare resources (due to good end-user experience), streaming services (video streaming) collect them. All other TCs (TCP real-time, interactive and background) are left with their minimum capacity (10\% of the TXOPs). These results are expectable given a preemptive policy, where the performance of the highest priority TC (conversational) is independent of the others [20].

The MOS (Fig 8a) directly depends on the service and the assigned resources. For this particular experimental conditions, VoIP traffic maintains good performance (MOS higher than $L M T_{1}=3.5$ ) up to 22 bidirectional calls, after which the conversational territory is saturated. The video streaming users perceive good subjective quality up to 10 voice conversations. Similarly, TCP video viewers experience a few (acceptable) buffer underruns up to 12 VoIP flows. Web browsing sessions have an almost ideal behavior for the whole range of voice calls (from 20 to 30 VoIP sources, MOS was around 3.4 3.5). This is due to the minimum capacity for the interactive traffic class, which is enough for 5 web browsing sessions due to the small amount of traffic generated (long reading periods). FTP only uses the minimum background capacity and the unused TXOPs. For this reason, its performance decreases as the VoIP load increases, performing well for up to 2 VoIP users and decreasing to a MOS $\approx 1.3$ (around $280 \mathrm{kbps}$ ).

The revenue metric (Fig 8.b) clearly shows that VoIP is the service with highest revenue efficiency (incomes per resource unit), followed by TCP video, FTP, video streaming and interactive with considerably smaller revenue. At the beginning, the revenue increases linearly with the number of voice flows, since their MOS is almost constant (around 3.8). When the conversational territory saturates and the VoIP performance decreases, its revenue also does since the resources are constant (and increment in the number of calls does not compensate the MOS reduction). The maximum revenue is 113 units, which is achieved with 22 calls.

Non-preemptive policy: In Fig. 9, the same metrics as for the previous case are shown for the Non-preemptive policy. The results are very dependent on the temporal evolution of the traffic sources, since it follows a first come, first served approach. This is the reason of this unpredictable behavior in the evolution of the MOS (Fig 9.a). To avoid this behavior, an admission control should start rejecting new flows when users start experiencing bad quality.

The maximum aggregated revenue (Fig 9.c) is 70 units, corresponding to 14 voice calls, VoIP being the highest contribution by far. The ranking of the services in terms of revenue is FTP and TCP video (with similar revenue), video streaming and finally web browsing (with almost no revenue).

MOS-driven Fairness policy: The results are shown in Fig. 10. In this case, resources are distributed so that different services will have similar quality (if there are not enough resources for having good performance). As shown in the Fig. 10.a, the MOS of VoIP, video streaming and FTP are very similar when the load increases and the MOS is lower than their respective $L M T_{i}$ $=3.5$. In fact, their territories vary in such a way that their MOS values are equalized. However, the MOS of the TCP video and web browsing services are higher, due to their minimum capacities. In this case the MOS of TCP video is always higher than 2.4, whereas web browsing achieves always good quality. A better performance for these services is obtained with low VoIP traffic because unused TXOPs are distributed among active flows [20].

However, fairness comes at a price. The global revenue (fig 10.c) is in fact reduced, since this policy does not prioritize the services with highest revenue efficiency. Its maximum value is 52 units, obtained for 12 VoIP stations.

Revenue Optimization policy: Fig. 11 summarizes the simulation results for the Revenue Optimization policy. This policy tries to maximize the income of the network operator.

It obtains similar aggregated revenue than the Preemptive policy (Fig 8.c) since VoIP (conversational) is the service with highest revenue efficiency. The variations in the territories try to maximize the incomes per resource unit, which leads to a prioritization of those services with higher revenue efficiency, i.e. firstly VoIP, then TCP video, FTP, video streaming and 
(a)

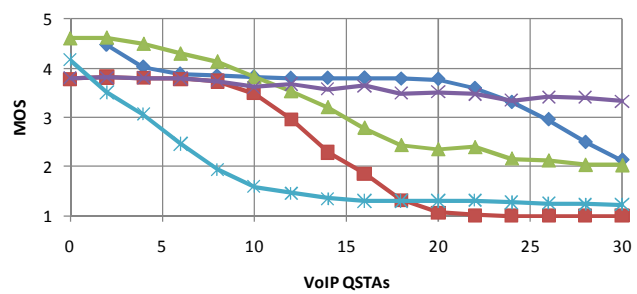

$\neg$ VolP $\rightarrow$-Video streaming $\longrightarrow$ TCP video $\multimap$ Web browsing $\rightarrow$ FTP

(b)

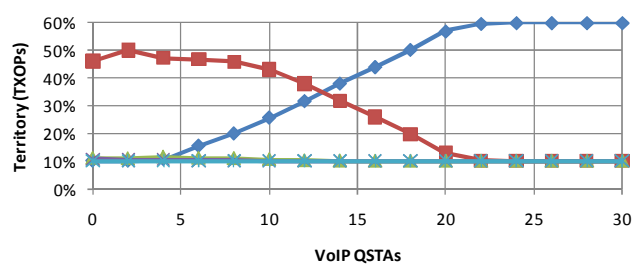

$\multimap$ CONVERSATIONAL $\rightarrow$ STREAMING $\quad \longrightarrow$ TCP REALTIME

*INTERACTIVE \#BACKGROUND

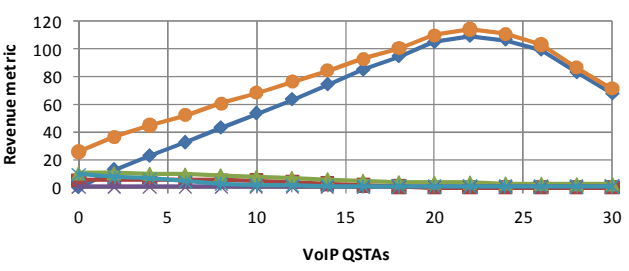

$\multimap$ CONVERSATIONAL $\rightarrow$ STREAMING $\quad \longrightarrow$ TCP REALTIME

*INTERACTIVE $\quad$ - BaCKGROUND $\rightarrow$ Aggregated

Figure 8. Results for the Preemptive policy.

(a)

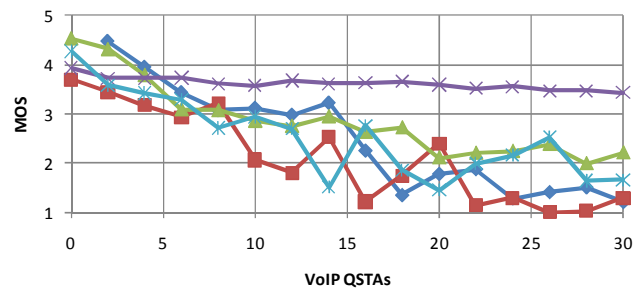

$\leadsto$ VolP $\rightarrow$-Video streaming $\leadsto$ TCP video $*$ Web browsing $\leadsto$ FTP

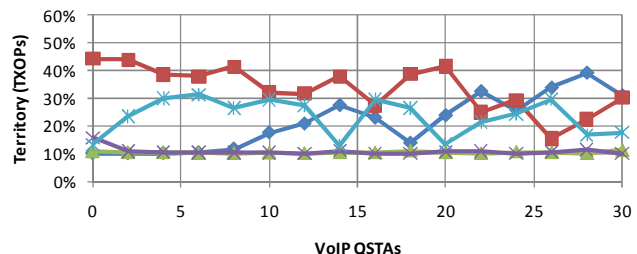

$\multimap$ CONVERSATIONAL $\because$-STREAMING $\quad \longrightarrow$ TCP ReAltIME *INTERACTIVE *BACKGROUND

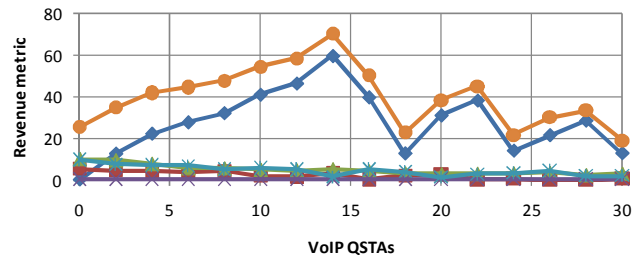

$\sim$ Conversational $\leadsto$ - STREAMING $\quad-$ TCP RealTIME

*interactive \#-Background $\rightarrow$ Aggregated

Figure 9. Results for the Non-preemptive policy. (a)

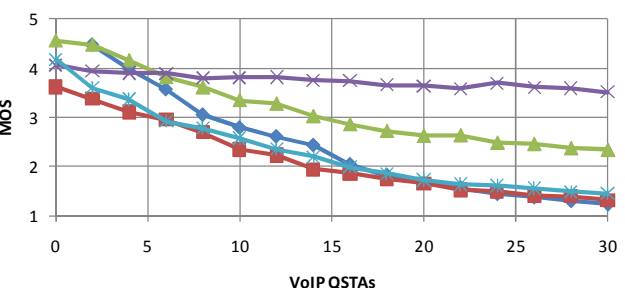

$\multimap$ VolP $\rightarrow$-Video streaming $\rightarrow$ TCP video $*$ Web browsing $\rightarrow$ FTP

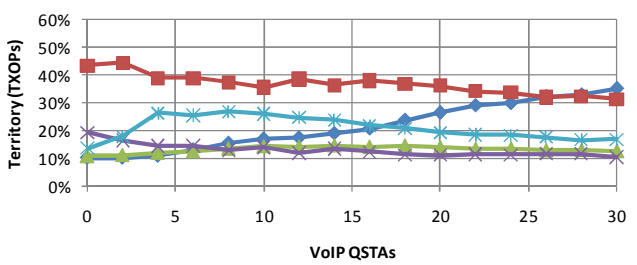

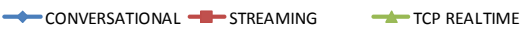

*interactive \#-Background

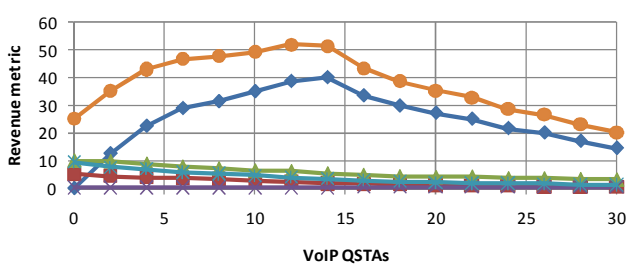

$\leadsto$ CONVERSATIONAL $\rightarrow$ STREAMING $\quad \longrightarrow$ TCP REALTIME

*INTERACTIVE *BACKGROUND $\rightarrow$ Aggregated

Figure 10. Results for the MOS-driven Fairness policy.

(a)
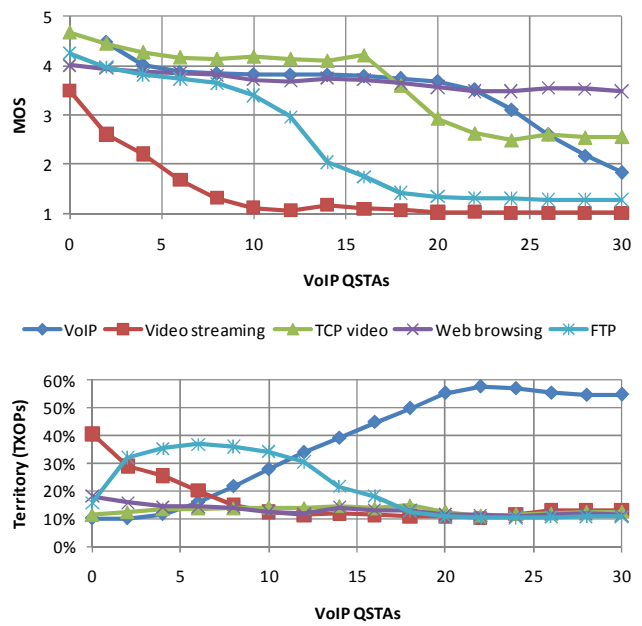

$\leadsto$ CONVERSATIONAL $\rightarrow$ - STREAMING $\rightarrow$ TCP RealtiME

*INTERActive *BAckground

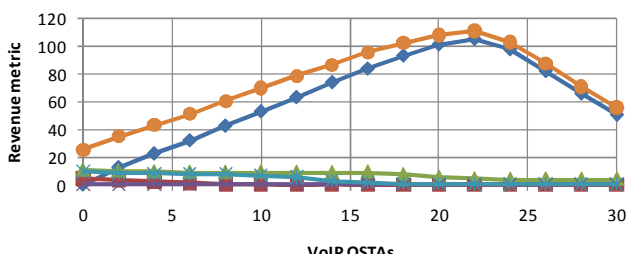

$\multimap$ COnVERSATIONAL $\multimap$-STREAMING $\quad$ TCP REALTIME

*INTERACTIVE $\quad$ *BACKGROUND - Aggregated

Figure 11. Results for the Revenue Optimization policy. 
finally web browsing. This greedy policy causes high variations in terms of MOS among the different traffic classes (Fig. 11.a) following the revenue distribution (Fig. 11.c), e.g. the quality is much lower for video streaming flows than for VoIP flows. In this scenario, the maximum benefit is 113 units when the voice calls are 22 , obtaining the highest revenue of the proposed policies.

As commented, the profit values are similar with this policy and with the Preemptive scheme. However, the introduction of other services (with different resource requirements) or different revenue factors for each TCs would lead to situations when this policy would maximize the revenue whereas the Preemptive scheme would not.

\section{CONCLUSIONS}

In this paper we present new resource sharing policies for a general framework [20] that allows the network operator to customize how resources are shared in an 802.11e network using the HCCA medium access mechanism.

This framework (called the territory method) splits the transmission opportunities into different pools. These pools contain the resources for different traffic classes (conversational, streaming, TCP real-time, interactive and background), i.e. sets of services with similar requirements. The size of the pools is governed by the end-user experienced quality (in terms of mean opinion score) and the selected policy.

Our proposal includes four different policies: Preemptive, Non-preemptive, MOS-driven Fairness and Revenue Optimization schemes. Our analysis has drawn, in the simulated scenario, the following conclusions. The Preemptive policy permits the prioritization of the services. The Non-preemptive approach distributes the resources depending on the temporal evolution of the demand, in a first come, first served fashion. Therefore, the resource allocation is not known a priori and it does not follow a predefined criterion. This is not the case of the MOS-driven Fairness policy, which allows the operator to equalize the subjective quality between traffic classes. Finally, the Revenue Optimization algorithm maximizes the profit of the network operator (with factors to customize the incomes from each service).

On top of these policies, the adjustment of a dedicated capacity for each traffic class guarantees a minimum performance for those services.

\section{REFERENCES}

[1] IEEE Std. 802.11e-2005, Medium Access Control (MAC) Quality of Service Enhancements, November 2005.

[2] G. Costanzi, "A Linux Kernel Based Implementation of 802.11e HCCA", master thesis available at http://utenli.lycos.it/giannicostanzi/my-projects.html.

[3] A. Grilo, M. Macedo, M. Nunes, "A Scheduling Algorithm for QoS Support in IEEE 802.11e Networks", IEEE Wireless Communications, pp. 36-43, June 2003.

[4] C. Cicconetti, L. Lenzini, E. Mingozzi, G. Stea, "Design and Performance Analysis of the Real-Time HCCA Scheduler for IEEE 802.11e WLANs", Computer Networks, vol. 51, issue 9, pp. 2311-2325, June 2007.

[5] C. Cicconetti, L. Lenzini, E. Mingozzi, G. Stea, "An Efficient Cross Layer Scheduler for Multimedia Traffic in Wireless Local Area
Networks with IEEE 802.11e HCCA", ACM MC2R, vol. 11, no. 3, pp. 31-46, July 2007.

[6] 3GPP TS 23.107 v8.0.0, Quality of Service (QoS) Concept and Architecture (Release 8), December 2008.

[7] 3GPP TS 23.203 v9.2.0, Policy and Charging Control Architecture (Release 9), September 2009.

[8] S. Khan, S. Duhovnikov, E. Steinbach, M. Sgroi, W. Kellerer, "Application-driven Cross-layer Optimization for Mobile Multimedia Communications using a Common Application Layer Quality Metric", in Proc. IWCMC'06, Vancouver, CA, July 3-6, 2006

[9] S. Khan, S. Duhovnikov, E. Steinbach, W. Kellerer, "MOS-Based Multiuser Multiapplication Cross-Layer Optimization for Mobile Multimedia Communication", Advances in Multimedia, vol. 2007.

[10] A. Saul, "Simple Optimization Algorithm for MOS-Based Resource Assignment", in Proc. of VTC Spring 2008, Marina Bay, Singapore, May 2008, pp.1766-1770.

[11] A. Saul, G. Auer, "Multiuser Resource Allocation Maximizing the Perceived Quality", EURASIP Journal on Wireless Communications and Networking, vol. 2009.

[12] P. Ameigeiras, J. J. Ramos-Munoz, J. Navarro-Ortiz, J. Ramiro-Moreno, J. M. Lopez-Soler, "QoE Evaluation of Scheduling Algorithms for NRT Services in LTE", in Proc. ICT-MobileSummit 2009, Santander, Spain.

[13] ITU-T Rec. P.800, Methods for Subjective Determination of Transmission Quality, August 1996.

[14] ITU-T Rec. G.107, The E-model, a Computational Model for Use in Transmission Planning, March 2005.

[15] J. Klaue, B. Rathke, A. Wolisz, "EvalVid - A Framework for Video Transmission and Quality Evaluation", in Proc. Performance TOOLS 2003, Urbana, Illinois, USA, September 2003, pp. 255-272.

[16] A. Khan, L. Sun, E. Ifeachor, "Content-Based Video Quality Prediction for MPEG4 Video Streaming over Wireless Networks", Journal of Multimedia, vol. 4, issue 4, pp. 228-239, August 2009.

[17] F. Sonnati, "Implementing a Dual-Threshold Buffering Strategy in Flash Media Server", http://www.adobe.com/devnet/flashmediaserver/articles/ fms_dual_buffering.html

[18] A. King, "The Average Web Page", October 2006, http://www.optimizationweek.com/reviews/average-web-page.

[19] Y. Sato, S. Kitano, S. Ata, I. Oka, "The Measurement of User's Subjective Assessment on Throughput Degradation in Bulk Data Transmission", in Proc. APNOMS 2008, October 2008, pp. 236-245.

[20] J. Navarro-Ortiz, P. Ameigeiras, J. J. Ramos-Munoz, J. M. Lopez-Soler, "An 802.11e HCCA scheduler with an end-to-end quality aware territory method", Computer Communications, vol. 32, issue 11, pp. 1281-1297, July 2009.

[21] A. Francini, F. Chiussi, "Minimum-latency dual-leaky-bucket shapers for packet multiplexers: theory and implementation", in Proc. IWQoS 2000, June 2000, pp. 19-28.

[22] Network Simulator 2, http://www.isi.edu/nsnam/ns.

[23] C. Cicconetti, L. Lenzini, E. Mingozzi, G. Stea, "A Software Architecture for Simulating IEEE 802.11e HCCA", in Proc. IPS MoMe 2005, Warsaw, Poland, March 2005, pp. 97-104.

[24] M. Andreozzi, G. Stea, C. Vallati, "A Framework for Large-scale Simulations and Output Result Analysis with ns-2", in Proc.QoSim 2009, Rome, Italy, March 2009.

[25] ITU-T Rec. P.59, Artificial Conversational Speech, March 1993.

[26] A. Bacioccola, C. Cicconetti, G. Stea, "User-level performance evaluation of VoIP using NS-2", in Proc. NSTools 2007, Nantes, France, October 2007, pp. 1-10.

[27] C. Ke, C. Shieh, W. Hwang, A. Ziviant, "An Evaluation Framework for More Realistic Simulations of MPEG Video Transmission", Journal of Information Science and Engineering, vol. 24, no. 2, pp. 425-440, March 2008.

[28] X. Cheng, C. Dale, J. Liu, "Statistics and Social Network of YouTube Videos", in Proc. IWQoS 2008, pp. 229-238, 2008.

[29] M. Molina, P. Castelli, G. Foddis, "Web traffic modeling: exploiting TCP connections' temporal clustering through HTML-REDUCE", IEEE Network, pp. 46-55, June 2000. 